\title{
QUANTITATIVE ESTIMATES ON JACOBIANS FOR HYBRID INVERSE PROBLEMS
}

G. Alessandrini, Department of Mathematics and Geosciences, University of Trieste, Trieste, Italy, alessang@units.it, V. Nesi, Department of Mathematics, Sapienza University of Rome, Rome, Italy, nesi@mat.uniroma1.it

We consider $\sigma$-harmonic mappings, that is mappings $U$ whose components $u_{i}$ solve a divergence structure elliptic equation $\operatorname{div}\left(\sigma \nabla u_{i}\right)=0$, for $i=1, \ldots, n$. We investigate whether, with suitably prescribed Dirichlet data, the Jacobian determinant can be bounded away from zero. Results of this sort are required in the treatment of the so-called hybrid inverse problems, and also in the field of homogenization studying bounds for the effective properties of composite materials.

Keywords: elliptic equations; Beltrami operators; hybrid inverse problems; composite materials.

In memoria di Alfredo.

\section{Introduction}

The appearance of coupled physics methods has provoked a sharp change of perspective in inverse boundary problems. The simultaneous use of different physical modalities to interrogate, through exterior measurements, a body whose interior parameters are unknown has enabled to single out interior functionals which carry useful, and possibly stable, information on the parameters of interest. Such methods are also known under the name of "hybrid inverse problems". Notable examples are the coupling of Magnetic Resonance with Electrical Impedance Tomography [1], Ultrasound and Electrical Impedance Tomography [2], Magnetic Resonance and Elastography [3]. To fix ideas, let us focus on Ultrasound Modulated Electrical Impedance Tomography. In EIT the goal is to determine the, possibly anisotropic, electrical conductivity $\sigma=\left\{\sigma_{i j}\right\}$ of a body $\Omega$ by repeated boundary measurements of voltage $\left.u\right|_{\partial \Omega}$ and current distribution $\sigma \nabla u \cdot \nu$ with $u$ solving the elliptic PDE

$$
\operatorname{div}(\sigma \nabla u)=0, \quad \text { in } \Omega
$$

As is well known [4], the stability is very weak and, in fact, in the anisotropic case, also non-uniqueness occurs [5]. By combining electrical measurements with ultrasound measurements it is possibile to focus on a tiny spot near any point $x \in \Omega$ and it has been shown by Ammari et al. [2] that one can detect the localized energy

$$
H(x)=\sigma \nabla u \cdot \nabla u(x) .
$$

If one repeats the experiments with different boundary voltages, it is possible to extract the functionals

$$
H_{i j}(x)=\sigma \nabla u_{i} \cdot \nabla u_{j}(x)
$$


where $u_{1}, \ldots u_{n}$ is an array of different solutions to (1). In Monard and Bal [6,7], it is shown how, from such functionals, one may obtain the conductivity $\sigma$ in a satisfactory stable fashion. The crucial point, however, is to be able to set up an array of boundary data $\phi_{1} \ldots, \phi_{n}$ and corresponding solutions $u_{1}, \ldots u_{n}$ in such a way that the functionals $H_{i j}$ are non degenerate.

In other words, calling $U: \Omega \rightarrow \mathbb{R}^{n}$, the mapping $U=\left(u_{1}, \ldots, u_{n}\right)$, which we shall designate " $\sigma$-harmonic" mapping, it is required that the Jacobian determinant det $D U$ does not vanish. And, furthermore, for the purpose of stability, a quantitative lower bound would be needed.

This is the main question that we wish to address in this note, which essentially stays behind all coupled physics problems mentioned above, and other inverse problems as well. The same issue showed up, for instance, in the field of groundwater transmissivity detection [8].

This kind of questions also arises in the branch of the homogenization theory which studies effective properties of composite materials. We give a brief outline here.

Indeed, the positivity of Jacobians of injective $\sigma$-harmonic mappings has attracted attention in several applications. In two dimensions, the first application of this positivity has been given in [9]. The long standing problem of improving the so-called Hashin Shtrikman bounds [10] for the effective conductivity of composite materials was addressed in that paper. The method used was based on ideas of Murat and Tartar [11] and Tartar [12], a reference not easy to find. We refer to [13] for a more complete treatment.

The bottom line is as follows. The question of interest, in the simplest not yet known at that time, case is the following. Three numbers $0<\sigma_{1}<\sigma_{2}<\sigma_{3}$, representing the conductivity of three isotropic materials, called the phases, three "volume fractions" $p_{1}, p_{2}$ and $p_{3}$, summing up to 1 and representing the area proportions of the phases and a $2 \times 2$ matrix $A$, parametrizing the affine boundary data, are given. Assume that $\sigma=\sum_{i=1}^{3} \chi_{i}(x) \sigma_{i}$ where $\chi_{i}$ represents the characteristic function of the set where $\sigma$ is equal to $\sigma_{i}$ times the identity matrix and $\frac{1}{|\Omega|} \int_{\Omega} \chi_{i}(x) d x=p_{i}, i=1,2,3$. Then one aims to determine a bound from below for the following quantity

$$
F(A)=\inf _{\chi_{1}, \chi_{2}, \chi_{3}} \inf _{U_{0} \in W_{0}^{1,2}(\Omega)} \frac{1}{|\Omega|} \int_{\Omega} \operatorname{Trace}\left[\left(D U_{0}(x)+A\right)^{T} \sigma(x)\left(D U_{0}(x)+A\right)\right] d x .
$$

The overall problem is non linear and actually is linked with the notion of quasi-convexity. More precisely, it computes the quasiconvexification at the matrix $A$, of a non-convex function of $D U$, with $U-A x \in W_{0}^{1,2}(\Omega)$. This function turns out to be the minimum of three quadratic functions, as shown by Kohn and Strang in [14]. However, for our purposes, it is important to note that the infimum over $U_{0}$ in (4) is attained exactly when $U(x)=U_{0}(x)+A x$ is the $\sigma$-harmonic mapping with affine boundary data given by $U=A x$ on $\partial \Omega$. Optimal lower bound for (4) were found by Kohn and Strang exactly exploiting the connection with the optimal bound for effective conductivity found by Murat and Tartar and, later, by Cherkaev and Lurie [15]. The optimality is restricted to the case when only two isotropic phases are present that is, only two materials are "mixed". For three or more phases, the methods based on compensated compactness gave suboptimal bounds. In this specific context, the compensated compactness method uses simply the constraint that the Jacobian determinant of the matrix $D U$ is a null-lagrangian. 
The classic strategy gives the so-called "Wiener bound", that is the harmonic mean bound. It is obtained considering the test fields $B$ in the class

$$
\mathcal{B}_{0}:=\left\{B \in L^{2}(\Omega): \frac{1}{|\Omega|} \int_{\Omega} B(x) d x=A\right\} .
$$

One obtains

$$
\begin{gathered}
F(A) \geq F_{0}(A):=\inf _{B \in \mathcal{B}_{0}} \frac{1}{|\Omega|} \int_{\Omega} \operatorname{Trace}\left[B(x)^{T} \sigma(x) B(x)\right] d x= \\
=\text { Trace }\left[A^{T}\left(\frac{1}{|\Omega|} \int_{\Omega} \sigma^{-1}(x) d x\right)^{-1} A\right] .
\end{gathered}
$$

Tartar's ideas, based on compensated compactness, in this simplified context lead to an improved bound (called the "translation bound" by G.W. Milton) obtained by considering the new test field in the class

$$
\mathcal{B}_{1}:=\left\{B \in L^{2}(\Omega): \frac{1}{|\Omega|} \int_{\Omega} B(x) d x=A, \frac{1}{|\Omega|} \int_{\Omega} \operatorname{det} B(x) d x=\operatorname{det} A\right\} .
$$

One obtains

$$
F(A) \geq F_{1}(A):=\inf _{B \in \mathcal{B}_{1}} \frac{1}{|\Omega|} \int_{\Omega} \operatorname{Trace}\left[B(x)^{T} \sigma(x) B(x)\right] d x .
$$

The computation is more involved than (5). This technique, however, gives an optimal answer in two dimensions, when specialized to the case of two-phase isotropic materials. When one deals with more than two phases this approach is no longer optimal. The results in [16] have the following corollary. Set

$\mathcal{B}_{2}:=\left\{B \in L^{2}(\Omega): \frac{1}{|\Omega|} \int_{\Omega} B(x) d x=A, \frac{1}{|\Omega|} \int_{\Omega} \operatorname{det} B(x) d x=\operatorname{det} A \operatorname{det} B \geq 0\right.$, a.e. in $\left.\Omega\right\}$.

One has

$$
F(A) \geq F_{2}(A):=\inf _{B \in \mathcal{B}_{2}} \frac{1}{|\Omega|} \int_{\Omega} \operatorname{Trace}\left[B(x)^{T} \sigma(x) B(x)\right] d x .
$$

In fact, in [9], it is proved that $F_{2}(A)>F_{1}(A)$, as soon as one deals with more that two isotropic phases, for suitable choices of the given parameters $p_{i}$ and matrices $A$. Later, new optimal microgeometries were found for multiphase materials in [17] and, using again the positivity of the Jacobian determinant, it was possible to prove their optimality according to a stricter criterion, see [18]. The key is exactly the universal bound given on the Jacobian determinant, which, in this context reads as the inequality $\operatorname{det} A \operatorname{det} B \geq 0$ in (6). In this context, it is highly desirable not to have any constraint on the regularity of the interfaces between phases. When $\sigma$ is non-symmetric, applications to composites have been 
given, for instance, in the context of the classic Hall effect by Briane and Milton [19,20]. Other applications have considered the problem of determining which electric fields are realizable by Briane, Milton, and Treibergs [21]. On the other hand one would like to have similar improvements in higher dimensions. Briane and Nesi [22] studied the case of laminates of high rank showing that, for these special microgeometries the positivity of the determinant of the "Jacobians" of the corrector matrix holds in any dimension. To explain the result in detail would require too long a digression. However, roughly speaking, one could expect that in higher dimensions, even for discontinuous $\sigma$ one could hope for the positivity of the Jacobian determinant if one makes assumptions on the "microgeometry". On the other hand, even in the very restricted setting of periodic boundary conditions, particularly adapted to composites, and even under the assumption of dealing with only two isotropic phases, there is no hope to control the sign of the Jacobian determinant of $\sigma$-harmonic mappings without further assumptions on the nature of the interfaces. One explicit example was provided by Briane, Milton and Nesi [23].

We now go back to the precise subject of the present paper. We pose the following problem. Problem 1.

Can we find Dirichlet data

$$
\Phi=\left(\phi_{1}, \cdots, \phi_{n}\right): \partial \Omega \rightarrow \mathbb{R}^{n}
$$

such that the corresponding solution mapping $U=\left(u_{1}, \cdots, u_{n}\right)$ is such that $\operatorname{det} D U$ is bounded away from zero independently of the conductivity $\sigma$ ?

Note that, in this context, it is essential that the choice of the boundary data is independent of $\sigma$, because $\sigma$ is the real unknown of the original inverse problem. As is easily understandable, some a-priori assumptions on $\sigma$, such as ellipticity, and some kind of regularity shall be needed.

Problem 1 has a different phenomenology depending on the space dimension. When $n=2$ the issue is more or less completely understood, whereas when $n=3$ or higher, various kinds of pathologies show up. A review of such pathologies and a discussion of the open issues when $n \geq 3$ shall be the object of Section 3 .

The principal aim of this note is to provide, when $n=2$, a quantitative lower bound on the Jacobian determinant under essentially minimal regularity assumptions. This is the content of our main Theorem 2 which is the new contribution of this paper to this subject.

We start reviewing the main known results in dimension $n=2$. It was proved in Bauman et al [16] that, if $\sigma$ is Hölder continuous, $\Omega$ has $C^{1, \alpha}$ boundary and $\Phi$ is a $C^{1, \alpha}$ diffeomorphism onto the boundary of a convex domain, then $\operatorname{det} D U>0$ everywhere. Note that in [16], only symmetric matrices $\sigma$ were explicitly considered, however, in view of classical results on two dimensional elliptic first order systems with Hölder coefficients see, for instance, [24] Appendix and also [25, Proposition 5.1], the result extends as well to the non-symmetric case. On the other hand, the present authors [26], proved that when $\sigma$ is merely $L^{\infty}$ and $\Phi$ is a homeomorphism onto the boundary of a convex domain, then $\operatorname{det} D U>0$ almost everywhere. In fact it was proved that, for every locally invertible, sense preserving, $\sigma$-harmonic mapping $U$ one has

$$
\log \operatorname{det} D U \in B M O
$$

and, subsequently [27], this result was improved to

$$
\operatorname{det} D U \in A_{\infty}
$$


that is the class of Muckenhoupt weights [28].

We recall that for purely harmonic mappings, Lewy's Theorem [29], states that for two-dimensional harmonic homeomorphisms, the Jacobian determinant cannot vanish at interior points. Hence, when $n=2$, harmonic homeomorphisms are, indeed, diffeomorphisms. However the Jacobian determinant may vanish at boundary points.

It is also worth mentioning that the convexity assumption on the target of the boundary mapping $\Phi$ is sharp, Choquet [30], Alessandrini and Nesi [31], if one wishes to have a condition expressed merely on the "shape" of the target and not on its parametrization.

Conversely, note that when no regularity is assumed on $\sigma$, the essential infimum of $\operatorname{det} D U$ on compact subsets of $\Omega$ might indeed be zero. In Section 2, an example, based on a well-known one by Meyers, is illustrated.

In the next Section 1 we shall prove a quantitative version of the result in [16]. The starting point relies on prescribing some quantitative assumption on the boundary data $\Phi$, when viewed as a parametrization of the boundary of the convex target, see Definitions 1, 2, 4 . The subsequent step consists on a quantitative lower bound of the modulus of the gradient of a scalar solution to equation (1), Theorem 1 . This estimate may be interesting on its own. Finally we state and prove our main result, Theorem 2.

\section{The Quantitative Bounds}

Let $\phi: \mathbb{R} \rightarrow \mathbb{R}$ be a $T$-periodic $C^{1}$ function. Let $\omega:[0, \infty) \rightarrow[0, \infty)$ be a continuous strictly increasing function such that $\omega(0)=0$.

Definition 1. Given $m, M \in \mathbb{R}, m<M$, we say that $\phi$ is quantitatively unimodal if there exists numbers $t_{1} \leq t_{2}<t_{3} \leq t_{4}<t_{1}+T$ such that

$$
\begin{array}{rlrl}
\phi(t) & =m & & t \in\left[t_{1}, t_{2}\right], \\
\phi(t) & =M & & t \in\left[t_{3}, t_{4}\right], \\
\phi^{\prime}(t) & \geq \min \left\{\omega\left(t-t_{2}\right), \omega\left(t_{3}-t\right)\right\}, & & t \in\left[t_{2}, t_{3}\right], \\
-\phi^{\prime}(t) & \geq \min \left\{\omega\left(t-t_{4}\right), \omega\left(t_{1}+T-t\right)\right\}, & t \in\left[t_{4}, t_{1}+T\right] .
\end{array}
$$

In the sequel we will refer to the quadruple $\{T, m, M, \omega\}$ as to the "character of unimodality" of $\phi$.

The concept of unimodality, but not this terminology, first appears in Kneser [32], when he proved Radò's conjecture [33] concerning the case of "purely" harmonic mappings. The terminology "unimodality" was introduced in this context by Leonetti and Nesi [34], following the work of Alessandrini and Magnanini [35]. A different terminology (almost two-to-one functions) has also been used for the same concept, Nachman, Tamasan and Timonov [36].

Let $\Gamma \subset \mathbb{R}^{2}$ be a simple closed curve parametrized by a $T$-periodic $C^{1}$ mapping

$$
\Phi: \mathbb{R} \rightarrow \mathbb{R}^{2}
$$

in such a way that $\Phi_{\mid[0, T)}$ is one-to-one.

Definition 2. We say that $\Gamma$ is quantitatively convex if for every $\xi \in \mathbb{R}^{2},|\xi|=1$ the function

$$
\phi_{\xi}=\Phi \cdot \xi
$$


is quantitatively unimodal and its character of unimodality is given by $\left\{T, m_{\xi}, M_{\xi}, \omega\right\}$ with $m_{\xi}, M_{\xi}$ such that $M_{\xi}-m_{\xi} \geq D$, for a given $D>0$.

In the sequel we will refer to the triple $\{T, D, \omega\}$ as to the "character of convexity" of $\Gamma$.

Remark 1. If $\Gamma$ is quantitatively convex then it is convex, that is, it is the boundary of a convex set $G$. In fact each tangent line to $\Gamma$ turns out to be a support line for $G$. The following Lemma provides a sufficient condition for quantitative convexity. Roughly speaking, it says that if $\Gamma$ is an appropriately parametrized $C^{2}$ simple closed curve with strictly positive curvature, then it is quantitatively convex in the sense of Definition 2, and the character of convexity can be computed in terms of the parametrization. Here, for the sake of simplicity, we have chosen the arc-length parametrization, because the main purpose of this Lemma is to provide a variety of examples, but we emphasize that in general, the character of convexity does depend on the parametrization of the curve and not only on its image.

We convene to denote by $J$ the matrix representing the counterclockwise rotation of 90 degrees

$$
J=\left(\begin{array}{cc}
0 & -1 \\
1 & 0
\end{array}\right)
$$

Lemma 1. Let $\Gamma$ be such that $\Phi \in C^{2}$ and:

$$
\begin{array}{ll}
\text { i) } & \left|\Phi^{\prime}\right|=1, \\
\text { ii) } & 0<\kappa \leq \Phi^{\prime \prime} \cdot J^{T} \Phi^{\prime} \leq K,
\end{array}
$$

then $\Gamma$ is quantitatively convex with character $\left\{|\Gamma|, \frac{1}{K}, \frac{2 \kappa}{\pi} t\right\}$.

Proof. Condition $i$ ) of Lemma 1, implies that $\Phi^{\prime}(t)=e^{i s(t)}, 0 \leq t \leq T$ and we may assume that $s(0)=0$. Without loss of generality we assume that $\Phi$ is orientation preserving. Then, by condition $i i$ ) of Lemma 1 , one has $0<\kappa \leq s^{\prime}(t) \leq K$. Picking, w.l.o.g., $\xi=e_{2}$,

$$
\phi_{\xi}(t)-\phi_{\xi}(0)=\int_{0}^{t} \sin (s(\tau)) d \tau \text {. }
$$

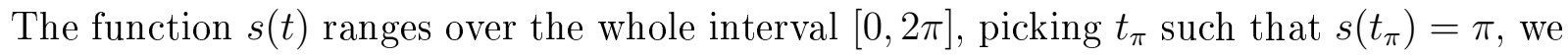
have

$$
\begin{aligned}
& M_{\xi}-m_{\xi}=\int_{0}^{t_{\pi}} \frac{\sin (s(\tau))}{s^{\prime}(\tau)} d s(\tau) \geq \frac{1}{K} \int_{0}^{\pi} \sin (s) d s=\frac{1}{K}, \\
& \phi_{\xi}(t)=\sin (s(t)), \\
& s(t)=\int_{0}^{t} s^{\prime}(\tau) d \tau \geq \kappa t, \\
& \phi_{\xi}(t) \geq \frac{2 \kappa}{\pi} t, \quad 0 \leq s(t) \leq \frac{\pi}{2} .
\end{aligned}
$$

Thus we may pick $D=\frac{1}{K}$ and $\omega(t)=\frac{2 \kappa}{\pi} t, t \geq 0$. 
We shall consider $\Omega$ a bounded simply connected domain in $\mathbb{R}^{2}$ with $C^{1, \alpha}$ boundary. In order to make precise the quantitative character of such regularity we introduce the following definition.

Definition 3. A domain $\Omega \subset \mathbb{R}^{2}$ is said to be of class $C^{1, \alpha}$ with constants $\rho_{0}, M_{0}$, positive and Hölder exponent $\alpha \in(0,1]$, if for any $P \in \partial \Omega$, there exist a rigid change of coordinates such that $P=0$ and we have

$$
\Omega \cap B_{\rho_{0}}(0)=\left\{x \in B_{\rho_{0}}(0): x_{2}>\psi\left(x_{1}\right)\right\},
$$

where $\psi:\left[-\rho_{0}, \rho_{0}\right] \rightarrow \mathbb{R}^{2}$ is a $C^{1, \alpha}$ function satisfying

$$
\psi(0)=\psi^{\prime}(0)=0
$$

and also

$$
\|\psi\|_{L^{\infty}\left(\left[-\rho_{0}, \rho_{0}\right]\right)}+\rho_{0}\left\|\psi^{\prime}\right\|_{L^{\infty}\left(\left[-\rho_{0}, \rho_{0}\right]\right)}+\rho_{0}^{1+\alpha} \sup _{\substack{x, x^{\prime} \in\left[-\rho_{0}, \rho_{0}\right] \\ x \neq x^{\prime}}} \frac{\left|\psi^{\prime}(x)-\psi^{\prime}\left(x^{\prime}\right)\right|}{\left|x-x^{\prime}\right|^{\alpha}} \leq M_{0} \rho_{0} .
$$

Definition 4. Given a $C^{1, \alpha}(\partial \Omega ; \mathbb{R})$ function $\phi$, we shall say that it is quantitatively unimodal, if considering the arclength parametrization of $\partial \Omega, x=x(s), 0 \leq s \leq T=|\partial \Omega|$, the periodic extension of the function $[0, T] \ni s \rightarrow \phi(x(s))$ is quantitatively unimodal with character $\{T, m, M, \omega\}$. For such a function $\phi$, we introduce the following closed arcs, possibly collapsing to a single point:

$$
\begin{aligned}
& \Gamma_{\min }=\{x \in \partial \Omega: \phi=m\}, \\
& \Gamma_{\max }=\{x \in \partial \Omega: \phi=M\} .
\end{aligned}
$$

Accordingly, a mapping $\Phi \in C^{1, \alpha}\left(\partial \Omega ; \mathbb{R}^{2}\right)$ shall be said quantitatively convex with character $\{T, D, \omega\}$ if the periodic extension of $\Phi(x(s))$ fulfils the conditions of Definition 2.

Let us consider $\sigma=\left\{\sigma_{i j}\right\}_{i, j=1,2}$ a, not necessarily symmetric, matrix of coefficients $\sigma_{i j}$ : $\bar{\Omega} \rightarrow \mathbb{R}$ satisfying the ellipticity condition

$$
\begin{gathered}
\sigma(x) \xi \cdot \xi \quad \geq K^{-1}|\xi|^{2}, \quad \text { for every } \xi \in \mathbb{R}^{2} \\
\sigma^{-1}(x) \xi \cdot \xi \geq K^{-1}|\xi|^{2}, \text { for every } \xi \in \mathbb{R}^{2}
\end{gathered}
$$

for given positive constant $K$, and also

$$
\left|\sigma_{i j}(x)-\sigma_{i j}\left(x^{\prime}\right)\right| \leq E\left|x-x^{\prime}\right|^{\alpha}, \quad \forall x, x^{\prime} \in \bar{\Omega},
$$

for given $\alpha, 0<\alpha \leq 1$ and $E>0$.

We shall consider the $W^{1,2}(\Omega)$ solution $u$ to the Dirichlet problem

$$
\begin{cases}\operatorname{div}(\sigma \nabla u)=0 & \text { in } \Omega \\ u=\phi & \text { on } \partial \Omega\end{cases}
$$

We recall that, in view of the classical regularity theory, $u$ in fact belongs to $C^{1, \beta}(\bar{\Omega})$, for some $\beta \leq \alpha$ and its norm is dominated by the $C^{1, \alpha}$-norm of $\phi$, modulo a constant which only depends on $\rho_{0}, M_{0}, K$ and $E$, with $\rho_{0}, M_{0}$ as in Definition 3 . 
Lemma 2. Let $\phi: \partial \Omega \rightarrow \mathbb{R}$ be quantitatively unimodal with character $\{|\partial \Omega|, m, M, \omega\}$ and assume that

$$
\left|\frac{d}{d s} \phi(x(s))-\frac{d}{d s} \phi\left(x\left(s^{\prime}\right)\right)\right| \leq E\left|s-s^{\prime}\right|^{\alpha}, \quad \forall s, s^{\prime} \in[0,|\partial \Omega|] .
$$

Then there exist $\kappa, \delta$ only depending on the character of unimodality (see Definitions 1, 4) and on $\alpha, E$, such that if

$$
x \in \bar{\Omega} \text { and } \operatorname{dist}\left(x, \Gamma_{\min } \cup \Gamma_{\max }\right) \leq \delta,
$$

then

$$
|\nabla u(x)| \geq \kappa
$$

Proof. Up to a $C^{1, \alpha}$ diffeomorphism, with constants only depending on $\rho_{0}, M_{0}$ and $|\partial \Omega|$, we may assume that $\Omega=B_{1}(0)$.

It is well known that in such new coordinates $u$ solves a new Dirichlet problem of type (19) with a new matrix of coefficients and new boundary data that, however, satisfy analogous assumptions with constants and parameters only depending on the same a-priori data. For the sake of not to overburn the notation we stick to the one of (19).

By the $C^{1, \beta}$ regularity of $u$, if $\operatorname{dist}\left(x, \Gamma_{\max }\right) \leq \eta$, then $u(x) \geq M-C \eta$ with $C>0$ only depending on the a-priori data.

Let us pick $\eta$ such that

$$
M-C \eta \geq \frac{M-m}{2}
$$

Hence, by Harnack's inequality [37],

$$
u(x)-m \geq C \frac{M-m}{2}>0, \text { for every } x \in B_{1-\eta}(0) .
$$

Here $C$ only depends on the a-priori data. By the version of the Hopf Lemma due to Finn and Gilbarg [38, Lemma 7], which applies to equations in divergence form, and Hölder continuous $\sigma$, we obtain

$$
|\nabla u(x)| \geq \kappa_{0}>0, \quad \forall x \in \Gamma_{\min },
$$

with $\kappa_{0}$ only depending on the a-priori data.

By $C^{1, \beta}$ regularity we have

$$
|\nabla u(x)| \geq \kappa_{0}-C \delta^{\beta} \quad \forall x \in \bar{\Omega} \text { such that } \operatorname{dist}\left(x, \Gamma_{\min }\right) \leq \delta
$$

Picking $\delta$ such that $C \delta^{\beta} \leq \frac{\kappa_{0}}{2}$, we obtain

$$
|\nabla u(x)| \geq \frac{\kappa_{0}}{2}>0, \quad \text { if } \operatorname{dist}\left(x, \Gamma_{\min }\right) \leq \delta .
$$

A symmetrical result applies in the neighborhood of $\Gamma_{\max }$.

Lemma 3. Under the same assumptions as in Lemma 2, there exists $r>0$ such that

$$
|\nabla u(x)| \geq L>0, \quad \forall x \in \bar{\Omega}, \quad \operatorname{dist}(x, \partial \Omega) \leq r .
$$


Here $L$ and $r$ are positive and only depend on the a-priori data.

Proof. If we pick $x \in \partial \Omega$, and write $x=x(s)$ such that $\operatorname{dist}\left(x, \Gamma_{\min } \cup \Gamma_{\max }\right) \geq \delta$, we have

$$
\mid \nabla u\left(x(s) \cdot x^{\prime}(s)|=| \frac{d}{d s} \phi(x(s) \mid \geq \omega(\delta) .\right.
$$

By $C^{1, \beta}$ regularity

$$
|\nabla u(x)| \geq \min \{\kappa, \omega(\delta)\}-C r^{\beta}, \quad \forall x \in \bar{\Omega} \text { such that } \operatorname{dist}(x, \partial \Omega)<r .
$$

Picking $r$ such that

$$
C r^{\beta}=\frac{1}{2} \min \{\kappa, \omega(\delta)\}
$$

the thesis follows.

Theorem 1. Let $\Omega$ be a simply connected domain, $C^{1, \alpha}$-regular with constants $\left\{\rho_{0}, M_{0}\right\}$ (see Definition 3). Let $\phi: \partial \Omega \rightarrow \mathbb{R}$ be quantitatively unimodal with given character $\{|\partial \Omega|, m, M, \omega\}$ (see Definitions 1, 4) and let it satisfy the Hölder condition (20). Let $\sigma=\left\{\sigma_{i j}(x)\right\}_{i, j=1,2}$ satisfy the ellipticity condition (17) and the Hölder bound (18). Let $u \in W^{1,2}(\Omega)$ be the solution of the Dirichlet problem (19).

Then there exists $C>0$, only depending on the a-priori data as above, such that

$$
|\nabla u(x)| \geq C>0, \text { for every } x \in \bar{\Omega} .
$$

Remark 2. Under stronger regularity assumptions, in particular assuming that $\sigma$ is Lipschitz continuous, a similar result was proven already in [40, Theorem 3.2].

Proof. As is well-known, there exists $\tilde{u} \in W^{1,2}(\Omega)$, called the stream function associated to $u$, which satisfies

$$
\nabla \tilde{u}=J \sigma \nabla u \text { everywhere in } \Omega, \quad J=\left(\begin{array}{cc}
0 & -1 \\
1 & 0
\end{array}\right) .
$$

Using complex notation $z=x_{1}+i x_{2}, f=u+i \tilde{u}$, the system (27) can be rewritten as

$$
f_{\bar{z}}=\mu f_{z}+\nu \bar{f}_{z} \quad \text { in } \Omega,
$$

where, the so called complex dilatations $\mu, \nu$ are given by

$$
\mu=\frac{\sigma_{22}-\sigma_{11}-i\left(\sigma_{12}+\sigma_{21}\right)}{1+\operatorname{Tr} \sigma+\operatorname{det} \sigma} \quad, \quad \nu=\frac{1-\operatorname{det} \sigma+i\left(\sigma_{12}-\sigma_{21}\right)}{1+\operatorname{Tr} \sigma+\operatorname{det} \sigma},
$$

and satisfy the following ellipticity condition

$$
|\mu|+|\nu| \leq \frac{K-1}{K+1}
$$

and, being $\sigma$ Hölder continuous, also $\mu$ and $\nu$ satisfy an analogous Hölder bound.

In [16], it is proven that $f$ is a $C^{1, \beta}$ diffeomorphism of $\bar{\Omega}$ onto $\overline{f(\Omega)}$. The lower bound obtained in Lemma 3, implies that, setting

$$
\Omega_{r}=\{x \in \Omega: \operatorname{dist}(x, \partial \Omega)>r\}
$$


$f: \bar{\Omega} \backslash \Omega_{r} \rightarrow \mathbb{C}$, is a bilipschitz homeomorphism with constants only depending on the a-priori data. We have identified $\mathbb{C}$ with $\mathbb{R}^{2}$ in the canonical way.

Hence $f(\Omega)$ is also a $C^{1, \beta}$ domain with constants controlled by the a-priori data. Note that also $|\partial(f(\Omega))|$ is controlled.

Let us denote $g=f^{-1}(w), w \in \mathbb{C}$. A straightforward calculation gives

$$
g_{\bar{w}}=-\nu(g) g_{w}-\mu(g) \overline{g_{w}} .
$$

In other words $g$ satisfies a Beltrami equation whose coefficients satisfy uniform ellipticity and Hölder continuity, with constants only depending on the a-priori data.

By standard interior regularity estimates, $g_{w}$ is bounded in $f\left(\Omega_{r}\right)$. Using (31), we have

$$
\left|g_{w}\right|^{2}-\left|g_{\bar{w}}\right|^{2} \leq C^{2} \quad \text { in } f\left(\Omega_{r}\right),
$$

which can be rewritten as

$$
\left|f_{w}\right|^{2}-\left|f_{\bar{w}}\right|^{2} \geq C^{-2} \text { in } \Omega_{r}
$$

which in turn implies

$$
|\nabla u| \geq C^{-1} \quad \text { in } \quad \Omega_{r} .
$$

Hence, in combination with Lemma 3 , the thesis follows.

Theorem 2. Let $\Omega$ and $\sigma$ be as in Theorem 1. Let $\Phi=\left(\phi_{1}, \phi_{2}\right): \partial \Omega \rightarrow \mathbb{R}^{2}$ be quantitatively convex, see Definitions 2, 4, with character $\{|\partial \Omega|, D, \omega\}$. Let $U=\left(u_{1}, u_{2}\right) \in W^{1,2}\left(\Omega ; \mathbb{R}^{2}\right)$ solve

$$
\begin{cases}\operatorname{div}\left(\sigma \nabla u_{i}\right)=0 & \text { in } \Omega, \\ u_{i}=\phi_{i} & \text { on } \partial \Omega .\end{cases}
$$

There exists $C>0$ only depending on the a-priori data such that

$$
U: \bar{\Omega} \rightarrow \overline{U(\Omega)} \subset \mathbb{R}^{2}
$$

is a $C^{1, \beta}$ diffeomorphism and

$$
\operatorname{det} D U \geq C^{2}>0 \text { in } \bar{\Omega} \text {. }
$$

Remark 3. A similar result, under slightly more restrictive hypotheses, has been recently proved by G.S. Alberti [39]. In fact the approach in [39] is based on estimates in [40,41] which require the Lipschitz regularity of $\sigma$. Conversely, under somewhat different regularity assumptions, quantitative upper bounds on the so-called dilatation of a $\sigma$-harmonic mapping $U$, that is the quotient

$$
\frac{\operatorname{Trace}\left(D U^{T} D U\right)}{2 \operatorname{det} D U}
$$

have been recently studied in [42, Theorems $3.1,3.4]$.

Proof. In [16], it is shown that $U$ is a orientation preserving diffeomorphism. The lower bound (35) remains to be proven. For any $\xi \in \mathbb{R}^{2},|\xi|^{2}=1$, we may apply Theorem 1 to

$$
u_{\xi}=U \cdot \xi
$$


and obtain

$$
|(D U) \xi|=\left|\nabla u_{\xi}\right| \geq C>0 \text { in } \bar{\Omega} .
$$

Or equivalently

$$
\left|D U^{T} D U \xi \cdot \xi\right|=\left|\nabla u_{\xi}\right|^{2} \geq C^{2}>0 \quad \text { in } \bar{\Omega},
$$

that is the eigenvalues $\lambda_{1}(x)$ and $\lambda_{2}(x)$ of the symmetric matrix $D U^{T}(x) D U(x)$ are uniformly bounded from below:

$$
\lambda_{i}(x) \geq C^{2}>0, \quad i=1,2, \quad \forall x \in \bar{\Omega} .
$$

Therefore

$$
(\operatorname{det} D U)^{2}=\lambda_{1}(x) \lambda_{2}(x) \geq C^{4}>0, \text { everywhere in } \bar{\Omega} .
$$

Since $U$ is sense preserving, one has $\operatorname{det} D U \geq C^{2}>0$ everywhere in $\Omega$.

Remark 4. Theorem 2, has some feature in common with the results in [31]. In the latter paper the authors consider harmonic mappings which are extensions of given Dirichlet data aiming for univalent solutions. A characterization is given, for the case when $U$ is a diffeomorphism up to the boundary of $\Omega$, in terms of the value of the Jacobian determinant on the boundary, so implicitly imposing constraints on the parametrization of the boundary of the image. One may wonder whether an assumption just on the shape of the target may suffice. This is not the case even in the purely harmonic case. Indeed one may exhibit a sequence $U_{n}$ of sense preserving, injective, harmonic mappings of the unit disk onto itself, fixing $U_{n}(0)=0$, such that det $D U_{n}(0) \rightarrow 0$ as $n \rightarrow+\infty$. The convergence holds uniformly on compact subsets of the unit disk. The limit harmonic mapping in not univalent. See [43, Section 4.1].

\section{Discontinuous Coefficients. An Example}

We elaborate on a well-known example by Meyers [44]. See also Leonetti and Nesi [34] for an application in a related context. For a fixed $\alpha>0$ we consider the symmetric matrix of coefficients

$$
\sigma(x)=\left(\begin{array}{cc}
\frac{\alpha^{-1} x_{1}^{2}+\alpha x_{2}^{2}}{x_{1}^{2}+x_{2}^{2}} & \frac{\left(\alpha^{-1}-\alpha\right) x_{1} x_{2}}{x_{1}^{2}+x_{2}^{2}} \\
\frac{\left(\alpha^{-1}-\alpha\right) x_{1} x_{2}}{x_{1}^{2}+x_{2}^{2}} & \frac{\alpha x_{1}^{2}+\alpha^{-1} x_{2}^{2}}{x_{1}^{2}+x_{2}^{2}}
\end{array}\right) .
$$

Is is a straightforward matter to check that its entries belong to $L^{\infty}$ and that $\sigma$ has eigenvalues $\alpha$ and $\alpha^{-1}$. Therefore $\sigma$ satisfies the uniform ellipticity condition (17) with ellipticity constant

$$
K=\max \left\{\alpha, \alpha^{-1}\right\}
$$

and $\sigma$ is discontinuous at $(0,0)$ (and only at $(0,0)$, when $\alpha \neq 1$ ). Let us denote

$$
\begin{aligned}
& u_{1}(x)=|x|^{\alpha-1} x_{1}, \\
& u_{2}(x)=|x|^{\alpha-1} x_{2} .
\end{aligned}
$$

A direct calculation shows that $u_{i} \in W^{1,2}\left(B_{1}(0)\right), i=1,2$ and that they solve the Dirichlet problem

$$
\left\{\begin{array}{llc}
\operatorname{div}\left(\sigma \nabla u_{i}\right)=0 & \text { in } & B_{1}(0), \\
u_{i}=x_{i} & \text { on } & \partial B_{1}(0) .
\end{array}\right.
$$


Note also that $f=u_{1}+i u_{2}$ is a quasiconformal mapping of $B_{1}(0)$ onto itself and it solves the Beltrami equation

$$
f_{\bar{z}}=\frac{\alpha-1}{\alpha+1} \frac{z}{\bar{z}} f_{z}
$$

Setting $U=\left(u_{1}, u_{2}\right)$, we compute

$$
\operatorname{det} D U=\left|f_{z}\right|^{2}-\left|f_{\bar{z}}\right|^{2}=\alpha|z|^{2(\alpha-1)} .
$$

Therefore det $D U$ vanishes at $(0,0)$ when $\alpha>1$, whereas, when $\alpha \in(0,1)$, it diverges as $z \rightarrow 0$.

\section{Mappings in Higher Dimensions. Examples and Open Problems}

The interior lower bound on $\operatorname{det} D U$ obtained in Theorem 2, has been achieved by methods which are intrinsically two-dimensional (the Beltrami equation). Only part of the result can be extended to higher dimensions.

For instance, with minor adaptations of the method developed in the Section 1, one can argue as follows.

Consider $\Omega \subset \mathbb{R}^{n}$, a bounded domain diffeomorphic to a ball of class $C^{1, \alpha}$ and with constants $\rho_{0}, M_{0}$ defined with the obvious slight adaptations of Definition 3 .

Let $\sigma=\left\{\sigma_{i j}\right\}_{i, j=1,2}$ be the matrix of coefficients and let it satisfy uniform ellipticity with constant $K$ as in (17) and Hölder continuity like in (18).

Let $G \subset \mathbb{R}^{n}$ be a convex body whose boundary $\Gamma$ is $C^{2}$ and having at each point principal curvatures bounded from below by $\kappa>0$.

Let $\Phi=\left(\phi_{1}, \phi_{2}, \cdots, \phi_{n}\right): \partial \Omega \rightarrow \Gamma$ be an orientation preserving diffeomorphism such that $\Phi, \Phi^{-1}$ are $C^{1, \alpha}$ with constant $E$. Let $U=\left(u_{1}, u_{2}, \cdots, u_{n}\right) \in W^{1,2}\left(\Omega ; \mathbb{R}^{n}\right)$ be the weak solution to

$$
\begin{cases}\operatorname{div}\left(\sigma \nabla u_{i}\right)=0 & \text { in } \Omega, \\ u_{i}=\phi_{i} & \text { on } \Omega, \\ i=1,2 \cdots, n . & \end{cases}
$$

Then, by the same arguments used in Section 1, we obtain.

Theorem 3. Under the above stated assumptions, there exists $\rho>0$ and $Q>0$ such that $U$ is a diffeomorphism of $\bar{\Omega} \backslash \Omega_{\rho}$ onto a neighborhood of $\Gamma$, within $\bar{G}$ and we have

$$
\operatorname{det} D U \geq Q \quad \text { in } \bar{\Omega} \backslash \Omega_{\rho} .
$$

We omit the proof.

When $n \geq 3$, there is no chance, under the kind of hypotheses stated above, to obtain a global lower bound on det $D U$. Evidence comes from a sequence of counterexamples that have been produced in a wide time span. A first illuminating example goes back to Wood [45] and has the amazing feature of being totally explicit. Wood displayed the following harmonic polynomial mapping from $\mathbb{R}^{3}$ onto $\mathbb{R}^{3}$ :

$$
U\left(x_{1}, x_{2}, x_{3}\right)=\left(u_{1}, u_{2}, u_{3}\right)=\left(x_{1}^{3}-3 x_{1} x_{3}^{2}+x_{2} x_{3}, x_{2}-3 x_{1} x_{3}, x_{3}\right)
$$

that is $U$ is a homeomorphism, but not a diffeomorphism because $\operatorname{det} D U=0$ on the plane $\left\{x_{1}=0\right\}$. 
Later Melas [46] provided an example of a three dimensional harmonic homeomorphism $U: B_{1}(0) \rightarrow B_{1}(0)$ such that $\operatorname{det} D U(0)=0$. Subsequently, Laugesen [47], showed that there exists homeomorphisms $\Phi: \partial B_{1}(0) \rightarrow \partial B_{1}(0)$ which are arbitrarily close to the identity in the sup-norm such that the mapping $U=\left(u_{1}, u_{2}, \cdots, u_{n}\right)$ solving

$$
\left\{\begin{array}{llr}
\Delta u_{i}=0 & \text { in } & B_{1}(0) \\
U=\Phi & \text { on } & \partial B_{1}(0) \\
i=1,2 \cdots, n . & &
\end{array}\right.
$$

is such that det $D U$ changes its sign somewhere inside $B_{1}(0)$. Such examples are especially striking because they show that in dimension $n \geq 3$ it seems difficult to find a universal rule to select Dirichlet data in such a way that the corresponding harmonic (or $\sigma$-harmonic mapping is invertible at a topological level (because it may reverse orientation!) and not only as a differentiable mapping.

One may wonder whether changing the topology of the boundary data may help. In the periodic case, obviously the harmonic functions are linear and this might have left the hope that, for variable coefficients, the periodic case may be better that the generic Dirichlet problem. However this is not the case. In [23], it was proved that, in dimension three, one can find a matrix $\sigma$ taking only two values, proportional to the identity matrix, and a periodic arrangement with a smooth interface, but such that the corresponding solution $U$ of the cell problem also reverses the orientation. The Jacobian determinant changes its sign in the interior of the (unit) cube of periodicity.

If, from the above examples, it seems that few chances are left of finding a universal criterion by which choosing Dirichlet data such that, for each $\sigma$ (although smooth) the corresponding $\sigma$-harmonic mapping $U$ has nondegenerate Jacobian, then a more reasonable goal would be to find a way to control, in term of the Dirichlet data, the set of points where the Jacobian may degenerate and possibly evaluate the vanishing rate at such points of degeneration.

This appears as a completely open problem, not at all easy as the following example by Jin and Kazdan [48] shows. Let $a \in C^{\infty}(\mathbb{R} ; \mathbb{R})$ and set

$$
\sigma(x)=\left(\begin{array}{ccc}
1 & a\left(x_{3}\right) & 0 \\
a\left(x_{3}\right) & 1 & 0 \\
0 & 0 & b\left(x_{3}\right)
\end{array}\right)
$$

with

$$
\left\{\begin{array}{ll}
a\left(x_{3}\right)=0 & \text { for } \quad x_{3} \leq 0 \\
a\left(x_{3}\right) \in\left(0, a_{0}\right) & \text { for } \quad x_{3}>0 \\
b\left(x_{3}\right)=\frac{1}{1-a^{2}\left(x_{3}\right)} & \text { for } \quad x_{3} \in \mathbb{R} .
\end{array} \text { with } \quad a_{0} \in(0,1)\right.
$$

We set

$$
U(x)=\left(x_{1}, x_{2},-x_{1} x_{2}+\phi\left(x_{3}\right)\right),
$$

where $\phi$ is chosen in such a way that

$$
\begin{cases}\left(b \phi^{\prime}\right)^{\prime}-2 a=0, & x_{3} \in \mathbb{R} \\ \phi\left(x_{3}\right)=0, & x_{3}<0\end{cases}
$$

It turns out that $\phi^{\prime}>0$ for $x_{3}>0$ and consequently

$$
\operatorname{det} D U= \begin{cases}\phi^{\prime}>0, & \text { for } x_{3}>0, \\ \phi^{\prime}=0, & \text { for } x_{3} \leq 0 .\end{cases}
$$

Вестник ЮУрГУ. Серия «Математическое моделирование 
This means that the Jacobian determinant of a $\sigma$-harmonic mapping does not fulfill the property of unique continuation (whereas this is the case for $|D U|^{2}=\operatorname{Trace}\left(D U^{T} D U\right)$ ). Hence the evaluation of the zero set of det $D U$ from boundary data might be troublesome.

Remark 5. The above example has some striking features. First of all note also that, letting $a_{0} \searrow 0$, we can make $\sigma$ as close as we want to the identity matrix. Moreover $U$ converges, uniformly on each compact subset of $\mathbb{R}^{3}$, to the harmonic polynomial mapping $U_{0}(x)=\left(x_{1}, x_{2},-x_{1} x_{2}\right)$.

We conclude by noticing that a limiting case of the above construction yields an example with a discontinuous, two-phase, $\sigma$ which is remarkable as well.

As before we pose

$$
\begin{gathered}
\sigma(x)=\left(\begin{array}{ccc}
1 & a\left(x_{3}\right) & 0 \\
a\left(x_{3}\right) & 1 & 0 \\
0 & 0 & b\left(x_{3}\right)
\end{array}\right), \\
\left\{\begin{array}{ll}
a\left(x_{3}\right)=0 & \text { for } x_{3} \leq 0, \\
a\left(x_{3}\right)=a_{0} & \text { for } x_{3}>0 \\
b\left(x_{3}\right)=\frac{1}{1-a^{2}\left(x_{3}\right)} & \text { for } \quad x_{3} \in \mathbb{R} .
\end{array} \text { with } a_{0} \in(0,1),\right.
\end{gathered}
$$

That is $\sigma$ is piecewise constant, namely

$$
\sigma(x)=\left(\begin{array}{ccc}
1 & 0 & 0 \\
0 & 1 & 0 \\
0 & 0 & 1
\end{array}\right) \quad \text { when } x_{3}<0 \text { and } \sigma(x)=\left(\begin{array}{ccc}
1 & a_{0} & 0 \\
a_{0} & 1 & 0 \\
0 & 0 & \frac{1}{1-a_{0}^{2}}
\end{array}\right) \text { when } x_{3}>0 \text {. }
$$

Again we pose

$$
U(x)=\left(x_{1}, x_{2},-x_{1} x_{2}+\phi\left(x_{3}\right)\right)
$$

where now $\phi$ is given by

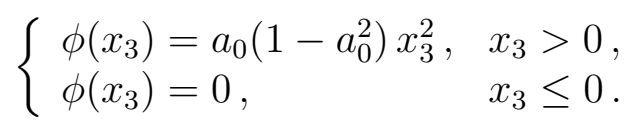

We obtain that $U$ is a $\sigma$-harmonic mapping with $C^{1,1}$ regularity and, analogously to the previous example, it satifies

$$
\operatorname{det} D U= \begin{cases}2 a_{0}\left(1-a_{0}^{2}\right) x_{3}>0, & \text { for } x_{3}>0 \\ 0, & \text { for } x_{3} \leq 0\end{cases}
$$

Acknowledgements. G. Alessandrini was supported by FRA2012 "Problemi Inversi", Università degli Studi di Trieste, V. Nesi was supported by PRIN Project 20102011 "Calcolo delle Variazioni".

\section{References}

1. Seo J.K., Woo E.J. Magnetic Resonance Electrical Impedance Tomography (MREIT). SIAM Rev., 2011, vol. 53, no. 1, pp. 40-68. DOI: 10.1137/080742932

2. Ammari H., Bonnetier E., Capdeboscq Y., Tanter M., Fink M. Electrical Impedance Tomography by Elastic Deformation. SIAM J. Appl. Math., 2008, vol. 68, no. 6, pp. $1557-1573$. DOI: $10.1137 / 070686408$ 
3. Honda N., McLaughlin J., Nakamura G. Conditional Stability for a Single Interior Measurement. Inverse Problems, 2014, vol. 30, no. 5, 055001. DOI: $10.1088 / 0266-5611 / 30 / 5 / 055001$

4. Mandache N. Exponential Instability in an Inverse Problem for the Schrödinger Equation. Inverse Problems, 2001, vol. 17, no. 5, pp. 1435-1444. DOI: 10.1088/0266-5611/17/5/313

5. Kohn R.V., Vogelius M. Identification of an Unknown Conductivity by Means of Measurements at the Boundary. Inverse Problems (New York), 1983, pp. 113-123, SIAMAMS Proc., 14, Amer. Math. Soc., Providence, RI, 1984.

6. Monard F., Bal G. Inverse Anisotropic Diffusion from Power Density Measurements in Two Dimensions. Inverse Problems, 2012, vol. 28, no. 8, 084001, 20 p.

7. Monard F., Bal G. Inverse Anisotropic Conductivity from Power Densities in Dimension. Comm. Partial Differential Equations, 2013, vol. 38, no. 7, pp. 1183-1207. DOI: $10.1080 / 03605302.2013 .787089$

8. Parravicini G., Giudici M., Morossi G., Ponzini G. Minimal a Priori Assignment in a Direct Method for Determining Phenomenological Coefficients Uniquely. Inverse Problems, 1995, vol. 11, no. 3, pp. 611-629. DOI: 10.1088/0266-5611/11/3/009

9. Nesi V. Bounds on the Effective Conductivity of Two-Dimensional Composites Made of $n \geq 3$ Isotropic Phases in Prescribed Volume Fraction: the Weighted Translation Method. Proceedings of the Royal Society of Edinburgh: Section A Mathematics, 1995, vol. 125, no. 6, pp. 1219-1239. DOI: $10.1017 /$ S0308210500030481

10. Hashin Z., Shtrikman S. A Variational Approach to the Theory of the Effective Magnetic Permeability of Multiphase Materials. Journal of Applied Physics, 1962, vol. 33, no. 10, pp. 3125-3131. DOI: 10.1063/1.1728579

11. Murat F., Tartar L. H-Convergence. Mathematical Modelling of Composite Materials. Progr. Nonlinear Differential Equations Appl., 1997, vol. 31, pp. 21-43.

12. Tartar L. Estimation de Coefficients Homogénéisés. Third International Symposium, December 5-9, 1977. Mathematics, Springer, Berlin, 1979, vol. 704, pp. 364-373.

13. Tartar L. The General Theory of Homogenization. Unione Matematica Italiana. Vol. 7. Springer-Verlag, Berlin; UMI, Bologna, 2009.

14. Kohn R.V., Strang G. Optimal Design and Relaxation of Variational Problems, II. Communications on Pure and Applied Mathematics, 1986, vol. 39, no. 2, pp. 139-182. DOI: $10.1002 /$ cpa.3160390202

15. Lurie K.A., Cherkaev A.V. Exact Estimates of Conductivity of Composites Formed by Two Isotropically Conducting Media Taken in Prescribed Proportion. Proceedings of the Royal Society of Edinburgh: Section A Mathematics, 1984, vol. 99, no. 1-2, pp. 71-87. DOI: $10.1017 /$ S030821050002597X

16. Bauman P., Marini A., Nesi V. Univalent Solutions of an Elliptic System of Partial Differential Equations Arising in Homogenization. Indiana Univ. Math. J., 2001, vol. 50, no. 2, pp. 747-757. DOI: 10.1512 /iumj.2001.50.1832

17. Albin N., Cherkaev A., Nesi V. Multiphase Laminates of Extremal Effective Conductivity in Two Dimensions. J. Mech. Phys. Solids, 2007, vol. 55, no. 7, pp. 1513-1553. DOI: $10.1016 /$ j.jmps.2006.12.003

18. Albin N., Conti S., Nesi V. Improved Bounds for Composites and Rigidity of Gradient Fields. Proc. R. Soc. Lond. Ser. A Math. Phys. Eng. Sci., 2007, vol. 463, no. 2084, pp. 2031-2048. DOI: $10.1098 /$ rspa.2007.1863 
19. Briane M., Milton G.W. Homogenization of the Three-Dimensional Hall Effect and Change of Sign of the Hall Coefficient. Arch. Ration. Mech. Anal., 2009, vol. 193, no. 3, pp. 715-736. DOI: $10.1007 / \mathrm{s} 00205-008-0200-\mathrm{y}$

20. Briane M., Milton G.W. An Antisymmetric Effective Hall Matrix. SIAM J. Appl. Math., 2010, vol. 70, no. 6, pp. 1810-1820. DOI: 10.1137/09075901X

21. Briane M., Milton G.W., Treibergs A. Which Electric Fields are Realizable in Conducting Materials. ESAIM: Mathematical Modelling and Numerical Analysis, 2014, vol. 48, no. 3, pp. 307-323. DOI: $10.1051 / \mathrm{m} 2 \mathrm{an} / 2013109$

22. Briane M., Nesi V. Is It Wise to Keep Laminating? ESAIM Control Optim. Calc. Var., 2004, vol. 10 , no. 4 , pp. $452-477$. (electronic) DOI: $10.1051 /$ cocv: 2004015

23. Briane M., Milton G.W., Nesi V. Change of Sign of the Corrector's Determinant for Homogenization in Three-Dimensional Conductivity. Arch. Ration. Mech. Anal., 2004, vol. 173, no. 1, pp. 133-150. DOI: 10.1007/s00205-004-0315-8

24. Bers L., Nirenberg L. On a Representation Theorem for Linear Elliptic Systems with Discontinuous Coefficients and Its Applications. Convegno Internazionale sulle Equazioni Lineari alle Derivate Parziali, Trieste, 1954. Edizioni Cremonese, Roma, 1955, pp. 111-140.

25. Bojarski B., D'Onofrio L., Iwaniec T., Sbordone C. G-Closed Classes of Elliptic Operators in the Complex Plane. Ricerche Mat., 2005, vol. 54, no. 2, pp. 403-432.

26. Alessandrini G., Nesi V. Univalent $\sigma$-Harmonic Mappings. Arch. Ration. Mech. Anal., 2001, vol. 158 , no. 2, pp. 155-171. DOI: $10.1007 /$ PL00004242

27. Alessandrini G., Nesi V. Beltrami Operators, Non-Symmetric Elliptic Equations and Quantitative Jacobian Bounds. Ann. Acad. Sci. Fenn. Math., 2009, vol. 34, no. 1, pp. 47-67.

28. Coifman R.R., Fefferman C. Weighted Norm Inequalities for Maximal Functions and Singular Integrals. Studia Math., 1974, vol. 51, pp. 241-250.

29. Lewy H. On the Non-Vanishing of the Jacobian in Certain One-To-One Mappings. Bull. Amer. Math. Soc., 1936, vol. 42, no. 10, pp. 689-692. DOI: 10.1090/S0002-9904-1936-06397-4

30. Choquet G. Sur un type de transformation analytique generalisant la representation conforme et definie au moyen de fonctions harmoniques. Bull. Sci. Math. (2), 1945, vol. 69, pp. 156-165.

31. Alessandrini G., Nesi V. Invertible Harmonic Mappings, Beyond Kneser. Ann. Scuola Norm. Sup. Pisa Cl. Sci. Series 5, 2009, vol. 8, no. 3, pp. 451-468.

32. Kneser H. Lösung der aufgabe 41. Jber. deutsch. math.-verein., 1926, vol. 35, pp. 123-124.

33. Radó T. Aufgabe 41. Jahresber. deutsch. math.-verein., 1926, vol. 35, p. 49.

34. Leonetti F., Nesi V. Quasiconformal Solutions to Certain First Order Systems and the Proof of a Conjecture of G.W. Milton. J. Math. Pures Appl., 1997, vol. 76, issue 2, pp. 109-124. DOI: $10.1016 /$ S0021-7824(97)89947-3

35. Alessandrini G., Magnanini R. Elliptic Equations in Divergence Form, Geometric Critical Points of Solutions, and Stekloff Eigenfunctions. SIAM J. Math. Anal., 1994, vol. 25, no. 5, pp. 1259-1268. DOI: $10.1137 /$ S0036141093249080

36. Nachman A., Tamasan A., Timonov A. Conductivity Imaging with a Single Measurement of Boundary and Interior Data. Inverse Problems, 2007, vol. 23, no. 6, pp. 2551-2563. DOI: 10.1088/0266-5611/23/6/017

37. Gilbarg D., Trudinger N.S. Elliptic Partial Differential Equations of Second Order. Grundlehren der mathematischen wissenschaften. Vol. 224. Berlin, Springer-Verlag, 1983. DOI: $10.1007 / 978-3-642-61798-0$ 
38. Finn R., Gilbarg D. Asymptotic Behavior and Uniqueness of Plane Subsonic Flows. Comm. Pure Appl. Math., 1957, vol. 10, issue 1, pp. 23-63. DOI: 10.1002/cpa.3160100102

39. Alberti G.S. Enforcing Local Non-Zero Constraints in PDEs and Applications to Hybrid Imaging Problems, 2014. arXiv:1406.3248.

40. Alessandrini G. Critical Points of Solutions of Elliptic Equations in Two Variables. Ann. Scuola Norm. Sup. Pisa Cl. Sci. Series 4, 1987, vol. 14, no. 2, pp. 229-256.

41. Alessandrini G. The Length of Level Lines of Solutions of Elliptic Equations in the Plane. Arch. Rational Mech. Anal., 1988, vol. 102, no. 2, pp. 183-191. DOI: 10.1007/BF00251498

42. Alessandrini G., Nesi V. Estimates for the Dilatation of $\sigma$-Harmonic Mappings. Rendiconti di Matematica e delle sue applicazioni, 2014, vol. 35, no. 3-4, pp. 215-225.

43. Duren P. Harmonic Mappings in the Plane. Cambridge University Press, 2004. DOI: 10.1017/CBO9780511546600

44. Meyers N.G. An $L^{p}$-Estimate for the Gradient of Solutions of Second Order Elliptic Divergence Equations. Ann. Scuola Norm. Sup. Pisa (3), 1963, vol. 17, pp. 189-206.

45. Wood J.C. Lewy's Theorem Fails in Higher Dimensions. Math. Scand., 1991, vol. 69, no. 2, pp. $166-166$.

46. Melas A.D. An Example of a Harmonic map between Euclidean Balls. Proc. Amer. Math. Soc., 1993, vol. 117, no. 3, pp. 857-859. DOI: 10.1090/S0002-9939-1993-1112497-9

47. Laugesen R.S. Injectivity Can Fail for Higher-Dimensional Harmonic Extensions. Complex Variables Theory Appl., 1996, vol. 28, no. 4, pp. 357-369. DOI: 10.1080/17476939608814865

48. Jin Z.R., Kazdan J.L. On the Rank of Harmonic Maps. Math. Z., 1991, vol. 207, no. 4, pp. 535-537. DOI: $10.1007 /$ BF 02571406

Received January 9, 2015

УДК 517.9

DOI: $10.14529 / \mathrm{mmp} 150302$

\title{
КОЛИЧЕСТВЕННЫЕ ОЦЕНКИ ЯКОБИАНА ДЛЯ ГИБРИДНОЙ ОБРАТНОЙ ЗАДАЧИ
}

\section{Д. Алессандрини, В. Нези}

\begin{abstract}
Рассматриваются $\sigma$-гармонические отображения, то есть отображения $U$ с компонентами $u_{i}$, являющимися решениями эллиптического уравнения $\operatorname{div}\left(\sigma \nabla u_{i}\right)=0$, для $i=1, \ldots, n$. Исследуется вопрос нахождения таких условий Дирихле, при которых Якобиан отделен от нуля. Результаты такого рода необходимы при решении так называемых гибридньх обратных задач, а также в теории усреднения границ для эффективных свойств композиционных материалов.

Ключевые слова: эллиптические уравнения; операторы Бельтрами; гибридные обратные задачи; композитные материалы.
\end{abstract}

Джованни Алессандрини, профессор, факультет математики и наук о Земле, Университет Триеста (г. Триест, Италия), alessang@units.it.

Винченцо Нези, профессор, факультет математики, Римский университет Ла Сапиенца (г. Рим, Италия), nesi@mat.uniroma1.it.

Поступила в редакиию 9 января 2015 г. 